\title{
ON THE WORK OF JEAN BOURGAIN IN NONLINEAR DISPERSIVE EQUATIONS
}

\author{
CARLOS E. KENIG
}

\begin{abstract}
In this brief note we survey a sample of the deep and influential contributions of Jean Bourgain to the field of nonlinear dispersive equations. Bourgain also made many fundamental contributions to other areas of partial differential equations and mathematical physics (as well as to a myriad of other areas in analysis, number theory, combinatorics, theoretical computer science, and more). Quoting the citation of the American Mathematical Society L. P. Steele Prize for Lifetime Achievement awarded to Bourgain in 2018, "Jean Bourgain is a giant in the field of mathematical analysis, which he has applied broadly and to great effect."

Jean Bourgain's contributions to mathematics will be remembered forever. Those of us who knew him will also remember his warmth, generosity, and graciousness.
\end{abstract}

\section{Nonlinear Dispersive EQUATIOns: \\ THE WELL-POSEDNESS THEORY BEFORE BOURGAIN}

The theory of nonlinear dispersive equations goes back to the nineteenth century, in connection with water waves in shallow water. The Korteweg-de Vries equation, which governs this phenomenon, was proposed by Boussinesq and by Korteweg and de Vries in the late nineteenth century as a way of explaining the discovery by Scott Russell (1835) of traveling waves. The generalized KdV equations ( $\mathrm{gKdV})_{k},(k=1$ being the Korteweg-de Vries equation) are

$$
(\mathrm{gKdV})_{k}\left\{\begin{array}{l}
\partial_{t} u+\partial_{x}^{3} u+u^{k} \partial_{x} u=0, x \in \mathbb{R}, \text { or } x \in \mathbb{T}, t \in \mathbb{R} \\
\left.u\right|_{t=0}=u_{0}(x)
\end{array}\right.
$$

(here, $\mathbb{T}$ and $\mathbb{T}^{d}$ are the one-dimensional ( $d$-dimensional) torus). Another example of nonlinear dispersive equations are the nonlinear Schrödinger equations (NLS),

$$
(\mathrm{NLS})\left\{\begin{array}{l}
i \partial_{t} u+\Delta u \pm|u|^{p-1} u=0, x \in \mathbb{R}^{d}, \text { or } x \in \mathbb{T}^{d} \\
\left.u\right|_{t=0}=u_{0}(x) .
\end{array}\right.
$$

When $d=1, p=3$, these equations model the propagation of wave packets in the theory of water waves. The equations also appear in nonlinear optics and in quantum field theory. These equations have a Hamiltonian structure and preserve mass and energy (although the energy may be negative). For both equations, the conserved mass is $\int\left|u_{0}\right|^{2}$, where the integral is over $\mathbb{R}^{d}$ or $\mathbb{T}^{d}$. For $(\mathrm{gKdV})_{k}$ the

Received by the editors September 2, 2020.

2020 Mathematics Subject Classification. Primary 35Q53, 35Q55.

The author's work is partially supported by NSF grant DMS- 1800082 . 
conserved energy is $E\left(u_{0}\right)=\int\left[\left(\partial_{x} u_{0}\right)^{2}-c_{k} u_{0}^{k+1}\right] d x$ and for (NLS) it is $E\left(u_{0}\right)=$ $\int\left[\left(\nabla_{x} u_{0}\right)^{2} \mp c_{p}\left|u_{0}\right|^{p+1}\right] d x$, where the integrals are over $\mathbb{R}^{d}$ or $\mathbb{T}^{d}$.

These equations are called dispersive because their linear parts are dispersive. Heuristically, the linear equations, when defined for $x \in \mathbb{R}^{d}$, are called dispersive, because the initial data gets spread out or dispersed by the evolution. (The linear equations can be solved by using Fourier's method.) Since the mass of the solution is constant (the $L^{2}$ norm is conserved), this requires the size of the linear solution to become small for large $t$, the so-called dispersive effect. Note that this is a feature of linear dispersive equations, the traveling wave solutions discovered by Russell do not have this property, they are purely nonlinear objects. Moreover, when $x \in \mathbb{T}^{d}$, there is no room for the solution to spread out, and the dispersive effect disappears.

Even though these equations were introduced in the nineteenth century and early twentieth century, their systematic study started much later. One of the first things to understand for such equations is well-posedness. Equations such as $(\mathrm{gKdV})_{k}$ or (NLS) are said to be locally well-posed in a space $B$ (with $u_{0} \in B$ ) if the equation has a unique solution $u$ (in a suitable sense) for $u_{0} \in B$, for some $T=T\left(u_{0}\right)$, $0 \leq t \leq T, u \in C([0, T] ; B)$, and the mapping $u_{0} \in B \rightarrow u \in C([0, T] ; B)$ is continuous. (That is to say, in analogy with ordinary differential equations, we have existence, uniqueness, and continuous dependence on the initial data.) If we can take $T=+\infty$, we say that the problem is globally well-posed. Since dispersive equations are (essentially) time reversible, we can replace $[0, T]$ by $[-T, T]$. Usually in this subject, the space $B$ is taken to be an $L^{2}$ based Sobolev space (or sometimes a weighted $L^{2}$ based Sobolev space, with power weights, in case we are working in $\mathbb{R}^{d}$ ). The reason for using $L^{2}$ based spaces as opposed to $L^{p}$ based spaces is the failure of estimates for $u_{0} \in L^{p}, p \neq 2$, in the associated linear problems. The first locally well-posed results used the analogy of these problems to classical hyperbolic ones, which led (by the classical energy method and its refinements and compactness arguments $([5,6]))$ to the local well-posedness of $(\mathrm{gKdV})_{k}$ in $H^{s}(\mathbb{R})$, for $s>\frac{3}{2}$, for $k=1,2, \ldots$, with the same result holding in $H^{s}(\mathbb{T})$, and to the local well-posedness of (NLS) in $H^{s}\left(\mathbb{R}^{d}\right)$, for $s>\frac{d}{2}$, with the same result holding in $H^{s}\left(\mathbb{T}^{d}\right)$. (In the case of (NLS) some restrictions on $p$ arise also, coming from the possible lack of smoothness of $\alpha \rightarrow|\alpha|^{p-1} \alpha$.) Here, for $f$ defined on $\mathbb{R}^{d}$, we set $\widehat{f}(\xi)=\int_{\mathbb{R}^{d}} e^{2 \pi i x \cdot \xi} f(x) d x, H^{s}\left(\mathbb{R}^{d}\right)=\left\{f: \int\left(1+|\xi|^{2}\right)^{s}|\widehat{f}(\xi)|^{2} d \xi<\infty\right\}$, and for $f$ defined on $\mathbb{T}^{d}$, we set $\widehat{f}(n)=\int_{\mathbb{T}^{d}} e^{2 \pi i x \cdot n} f(x) d x, n \in \mathbb{Z}^{d}$, and $H^{s}\left(\mathbb{T}^{d}\right)=$ $\left\{f: \sum_{n \in \mathbb{Z}^{d}}|\widehat{f}(n)|^{2}\left(1+|n|^{2}\right)^{s}<\infty\right\}$. An inspection of these proofs shows that dispersive properties of $\left(\partial_{t}+\partial_{x}^{3}\right)$ or of $\left(i \partial_{t}+\Delta\right)$ are not used at all in the case of $\mathbb{R}^{d}$, and hence they remain valid for the case of $\mathbb{T}^{d}$. Particular cases of $(\mathrm{gKdV})_{k}$ and (NLS) are closely connected to complete integrability, a theory which was first developed largely in this regard [1. These are the cases $k=1,2$ in $(\mathrm{gKdV})_{k}$ and $p=3, d=1$ in (NLS). The applicability of this method initially required a high order of differentiability of the data $u_{0}$ and, in the case $x \in \mathbb{R}$, a fast decay of $u_{0}$. More recently, this has been greatly improved (see [41, 42, 553) but still only applies to a few specific cases.

In the late 1970s and early 1980s, the pioneering works of Ginibre and Velo ([32, [33], 34]) and Kato [45, through the use of important new advances in harmonic analysis (83], 86]), led to low regularity locally well-posed and globally well-posed results for (NLS) in $\mathbb{R}^{d}$, culminating with the definitive results of Tsutsumi [85] and Cazenave and Weissler 22]. This approach exploited the dispersive properties of 
$\left(i \partial_{t}+\Delta\right)$ and the connection with the restriction problem for the Fourier transform (discovered and formulated in the visionary work of E. M. Stein (see [81]) uncovered by Segal [78] and Strichartz [83]).

More precisely, the solution of the initial value problem,

$$
\text { (LS) }\left\{\begin{array}{l}
i \partial_{t} u+\Delta u=0, x \in \mathbb{R}^{d}, t \in \mathbb{R} \\
\left.u\right|_{t=0}=u_{0}(x)
\end{array}\right.
$$

is given by

$$
\left.\widehat{u}(\xi, t)=e^{i t|\xi|^{2}} \widehat{u}_{0}(\xi)=\left(e^{i t \Delta} u_{0}\right) \hat{(\xi}\right)
$$

or

$$
u(x, t)=\frac{c_{d}}{|t|^{\frac{d}{2}}} \int_{\mathbb{R}^{n}} e^{i|x-y|^{2} / 4 t} u_{0}(y) d y .
$$

The second formula gives that for $u$ solving (LS),

$$
|u(x, t)| \leq \frac{c_{d}}{|t|^{\frac{d}{2}}}\left\|u_{0}\right\|_{L^{1}}
$$

which clearly shows the dispersive effect mentioned earlier. The relevant restriction problem here is the one to the paraboloid that equals $\left\{\left(\xi,|\xi|^{2}\right): \xi \in \mathbb{R}^{d}\right\} \subset \mathbb{R}^{d+1}$. In this case we have the restriction inequality

$$
\left(\int\left|\widehat{f}\left(\xi,|\xi|^{2}\right)\right|^{2} d \xi\right)^{\frac{1}{2}} \lesssim\|f\|_{L^{\frac{2(d+2)}{d+4}}\left(\mathbb{R}^{d+1}\right)}
$$

for $f \in \mathscr{S}\left(\mathbb{R}^{d+1}\right)$ (see [83, 86]). The connection with (LS) is that the dual inequality to (2) is the extension inequality, which gives, from the first formula for the solution $u$ of (LS), the estimate

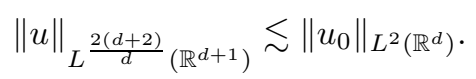

Now, to solve (NLS), one needs to solve (by Duhamel's principle) the equation (with the notation $\left.e^{i t \Delta} u_{0}=S(t) u_{0}\right)$ )

$$
u(t)=S(t) u_{0} \pm \int_{0}^{t} S\left(t-t^{\prime}\right)|u|^{p-1} u\left(t^{\prime}\right) d t^{\prime} .
$$

This is solved by using the contraction mapping principle on spaces constructed exploiting the estimate (2) and related ones (32, 33, [34, [45]).

The result of Cazenave and Weissler 22] follows.

Theorem 1.1. Assume that $u_{0} \in H^{s}\left(\mathbb{R}^{d}\right), s \geq 0, s \geq s_{0}$, where $p-1=\frac{4}{d-2 s_{0}}$. Assume also that $p-1>[s]+1$ if $p-1 \notin 2 \mathbb{Z}^{\star}$, where $[s]$ is the greatest integer smaller than s. Then (NLS) is locally well-posed for $t \in[-T, T]$. In the subcritical case $s>s_{0}$, we can take $T=T\left(\left\|u_{0}\right\|_{H^{s}}\right)$; in the critical case $s=s_{0}, T=T\left(u_{0}\right)$.

This approach, relying on the estimates (1) and (3) uses crucially the dispersive properties of $\left(i \partial_{t}+\Delta\right)$ in $\mathbb{R}^{d}$, and hence it does not apply to $\mathbb{T}^{d}$. On the other hand on $\mathbb{R}^{d}$ it yields essentially optimal results in terms of the values of $s$ when $B=H^{s}\left(\mathbb{R}^{d}\right)$, which greatly improve the results obtained by the energy method described earlier.

There are several motivations for hoping to have low regularity well-posedness results for $(\mathrm{gKdV})_{k}$ and (NLS). The first one is that if one can obtain local wellposedness at the regularity level given by the conserved mass or the conserved energy with time of existence $T=T\left(\left\|u_{0}\right\|\right)_{L^{2}}$, or $T=T\left(\left\|u_{0}\right\|_{H^{1}}\right)$, one can use the 
a priori control given by the conserved quantity to obtain global well-posedness, simply by iterating the local result. Another motivation is the belief that since for the associated linear problem we have well-posedness in $H^{s}$, for any $s$, the threshold $\bar{s}$ for the nonlinear problem, gives information on the nonlinear effects present in the problem. We will see later another motivation, at very low regularity levels, stemming from the connection with quantum field theory, and giving global well-posedness for generic data. Turning to the low regularity local well-posedness theory for $(\mathrm{gKdV})_{k}$, the new difficulty is the fact that the nonlinear term contains a derivative, which needs to be recovered. One might think that the fact that $\left(\partial_{t}+\partial_{x}^{3}\right)$ has a stronger dispersive effect (we have, for instance, the bound $|u(x, t)| \lesssim \frac{1}{t^{1 / 3}}\left\|u_{0}\right\|_{L^{1}}$ for the linear solution, which is stronger for small $t$ than the $\frac{1}{t^{1 / 2}}$ we get for (LS), $d=1$ ) would compensate for the derivative in the nonlinearity, but this is not obviously the case. Kato (43, 44) found a local smoothing effect for solutions of $(\mathrm{gKdV})_{k}$ which allowed, when $x \in \mathbb{R}$, for control a priori, with $u_{0} \in L^{2}(\mathbb{R})$ quantities like $\int_{j}^{j+1} \int_{0}^{1}\left(\partial_{x} u(x, t)\right)^{2} d x d t, j \in \mathbb{Z}$, uniformly in $j$, but this only gave rise to weak solutions with $L^{2}$ data and did not give uniqueness or continuous dependence on the data. This was also restricted to $x \in \mathbb{R}$, since such an estimate in $\mathbb{T}$ would contradict time reversibility and conservation of mass. In the 1980s and early 1990s, in a joint project with G. Ponce and L. Vega, we developed a new approach to the low regularity local and global well-posedness theory (for $x \in \mathbb{R})$ for $(\mathrm{gKdV})_{k}$, which in the case $k \geq 4$ gave essentially optimal (in some sense) results $(4$, 51]). This was also based on the contraction mapping theorem, and it used tools from harmonic analysis. In addition to the analogues of the extension inequality (3) (with $\left(\xi,|\xi|^{2}\right)$ being replaced by $\left(\xi, \xi^{3}\right)$ ), we used a sharp form (for linear equations) of the Kato local smoothing estimate, introduced in [30, 82, [87, as well as an analogue of the maximal function estimate introduced in [21] and motivated by statistical mechanics (see also [31, [87]). The combination of these two estimates allowed us to control well the nonlinear term $u^{k} \partial_{x} u$. In addition we also applied the multilinear harmonic analysis tools developed by Coifman and Meyer (23], 24]). This was all completely tied to dispersion and was totally dependent or the fact that $x \in \mathbb{R}$. A sample result obtained, for $\operatorname{KdV}(k=1)$, follows.

Theorem $1.2(49])$. Let $s>\frac{3}{4}, u_{0} \in H^{s}(\mathbb{R})$. Then $\exists T=T\left(\left\|u_{0}\right\|_{H^{s}}\right)$ and a space $X_{T}^{s} \subset C\left([-T, T] ; H^{s}\right)$, such that $K d V$ has a unique solution $u \in X_{T}^{s}$, which depends continuously on $u_{0}$.

The space $X_{T}^{s}$ is constructed by using the estimates mentioned earlier, namely the sharp local smoothing estimate, the maximal function estimate, and the variants of the extension estimate. One then proves the result by the contraction mapping principle in the space $X_{T}^{s}, T=T\left(\left\|u_{0}\right\|_{H^{s}}\right)$, showing that the mapping $\Phi_{u_{0}}(u)=$ $W(t) u_{0}+\int_{0}^{t} W\left(t-t^{\prime}\right)\left(u \partial_{x} u\right)\left(t^{\prime}\right) d t^{\prime}$ has a fixed point in $X_{T}^{s}$, where $\widehat{W(t) f}(\xi)=$ $e^{i t \xi^{3}} \widehat{f}(\xi)$.

Remark 1 . In a certain sense the approach was sharp: if we have a space $X_{T}^{s}$ such that $\forall u_{0} \in H^{s}(\mathbb{R})$, the linear solution $W(t) u_{0}$ belongs to $X_{T}^{s}$, and such that, for all $v, w \in X_{T}^{s}$ we have $v \partial_{x} w \in L_{\text {loc }}^{1}(\mathbb{R})$, then $s \geq \frac{3}{4}$.

At this point, we had no idea how to improve the results for $k=1,3$ (the $k=2$ result in [49] was also optimal, as was shown in [51), or how to do anything other than the $s>\frac{3}{2}$ result given by the energy method in the case $x \in \mathbb{T}$. 


\section{Bourgain's TRANSFORMATIVE WORK \\ ON THE WELL-POSEDNESS THEORY OF DISPERSIVE EQUATIONS}

In the spring of 1990, I gave a lecture on the work (then in progress) in [49, and E. Speer was in the audience. He asked me the following question: Consider the quintic (NLS) on $\mathbb{T}$ :

$$
\left\{\begin{array}{l}
i \partial_{t} u+\Delta u \pm|u|^{4} u=0, x \in \mathbb{T}, t \in \mathbb{R} \\
\left.u\right|_{t=0}=u_{0}(x) \in H^{s}(\mathbb{T}) .
\end{array}\right.
$$

Is this problem well-posed for $s<\frac{1}{2}$ ?

I knew that the energy method gave $s>\frac{1}{2}$, that complete integrability did not apply and that the methods I developed with Ponce and Vega, which relied on dispersion, did not apply. Speer explained the reason for the question, which was in connection with the work [56] of Lebowitz, Rose, and Speer, in which they had constructed a Gibbs measure associated to the problem (5). The points that the authors of [56] were concerned with were that the measure they constructed used the periodic setting crucially, and that the support of the measure was contained in very low regularity spaces. So, they wanted to have a flow for (15), in the support of the Gibbs measure, which kept the Gibbs measure invariant. If so, a byproduct of all this would be that for data in the support of the measure, local-in-time existence could be globalized in time, similarly to the arguments in the presence of conserved quantities that we saw before. I told Speer that I felt that the question was very hard, and that I thought the person who could make progress in it, and would probably be interested in the problem, was Jean Bourgain! Bourgain did get interested and resolved completely the Lebowitz-Rose-Speer questions. In doing so, he transformed the theory of nonlinear dispersive equations, starting with his papers [7], 8], 9]. Moreover, he continued making fundamental contributions to all aspects of this theory, and he transformed not only the well-posedness theory and created the probabilistic theory suggested by [10, [11, and [56], but also many other central areas in the field. Let me now turn to Bourgain's papers [7], 8], in which he made his first groundbreaking contributions to the well-posedness theory. These works address the following two fundamental questions:

1. How do we prove low regularity well-posedness results for (NLS) and $(\mathrm{gKdV})_{k}$ for $x \in \mathbb{T}^{d}$ ?

2. How do we improve the well-posedness results on $(\mathrm{KdV})$ on $\mathbb{R}$ ?

It turns out that in solving the first question, Bourgain also found the path to solving the second one. Also, once the first question was solved, Bourgain turned to the Gibbs measure questions from [56, in [10], [11, settling them and extending their scope, as we shall see below. We thus turn to (NLS) on $\mathbb{T}^{d}$, and we will concentrate on Bourgain's results for $d=1,2$, which are the most relevant to our exposition.

Theorem 2.1 ([7]).

(i) (NLS) is locally well-posed in $H^{s}(\mathbb{T})$, for $s \geq 0, p-1<\frac{4}{1-2 s}$. Thus, for $p-1=4,(\mathrm{NLS})$ is locally well-posed in $H^{s}(\mathbb{T})$ for all $s>0$.

(ii) (NLS) is locally well-posed in $H^{s}\left(\mathbb{T}^{2}\right)$, for $p-1=2, s>0$.

Compared with corresponding results in $\mathbb{R}, \mathbb{R}^{2}$ that we discussed earlier, one key difficulty is the lack of a dispersive effect. Another difficulty is that in the periodic 
case, the Fourier transform, in the solution of the associated linear problem, is replaced by Fourier series, leading to exponential sums that are much more difficult to estimate than integrals. For instance, the operator $e^{i t \Delta} u_{0}=S(t) u_{0}$ now takes the form

$$
S(t) u_{0}(x)=\sum_{n \in \mathbb{Z}^{d}} e^{i\left(x n+t|n|^{2}\right)} \widehat{u}_{0}(n) .
$$

The proof of Theorem 2.1 proceeds by using the contraction mapping principle. The first step is to find estimates that replace the inequality (3), crucial in the case of $\mathbb{R}^{d}$, which is proved using oscillatory integral estimates. Bourgain achieved this by using analytic number theory, and the results that he obtained in doing this have independent interest in analytic number theory. As a sample let me mention two such estimates:

(a)

$$
\left\|\sum_{n \in \mathbb{Z},|n| \leq N} a_{n} e^{i\left(n x+n^{2} t\right)}\right\|_{L^{6}\left(\mathbb{T}^{2}\right)} \lesssim N^{\varepsilon}\left(\sum\left|a_{n}\right|^{2}\right)^{\frac{1}{2}}, \forall \varepsilon>0,
$$

which is used in Theorem 2.1(i) and

(b)

$$
\left\|\sum_{n \in \mathbb{Z}^{2},\left|n_{1}\right| \leq N,\left|n_{2}\right| \leq N} a_{n} e^{i\left(n x+|n|^{2} t\right)}\right\|_{L^{4}\left(\mathbb{T}^{3}\right)} \lesssim N^{\varepsilon}\left(\sum_{n \in \mathbb{Z}^{2}}\left|a_{n}\right|^{2}\right)^{\frac{1}{2}}, \forall \epsilon>0,
$$

which is used in Theorem 2.1(ii).

Their proof uses the argument of Tomas ([86]) in the proof of the restriction inequality combined with the major arc description of exponential sums (due to Vinogradov) and number theoretic arguments inspired by Weyl-type lemmas 88. The second main contribution of Bourgain here is the introduction of new function spaces in which to apply the contraction mapping principle.

For $K, N$ positive integers, consider

$$
\Lambda_{K, N}=\left\{\zeta=(\xi, \lambda) \in \mathbb{Z}^{d} \times \mathbb{R}: N \leq|\xi| \leq 2 N \text { and } K \leq\left.|\lambda-| \xi\right|^{2} \mid \leq 2 K\right\} .
$$

For a function $u$ in $L^{2}\left(\mathbb{T}^{d} \times \mathbb{R}\right)$, let

$$
u(x, t)=\sum_{\xi \in \mathbb{Z}^{d}} \int \widehat{u}(\zeta) e^{2 \pi i(\xi x+t \lambda)} d \lambda,
$$

and define $\|u\| \|_{s}=\sup _{K, N}(K+1)^{\frac{1}{2}}(N+1)^{s}\left(\int_{\Lambda_{K, N}}|\widehat{u}(\zeta)|^{2} d \zeta\right)^{\frac{1}{2}}$.

Fixing an interval of $t$ in $[-\delta, \delta]$, one considers the restriction norm

$$
\|u\|_{X^{s}}=\inf \|\tilde{u}\|_{s},
$$

where the infimum is taken over all $\tilde{u}$ coinciding with $u$ in $[-\delta, \delta]$, and it shows that the integral equation has a solution in $X^{s}$, for small $\delta$, by (4), now on $\mathbb{T}^{d}$, using the contraction mapping theorem. This applies to (i) and (ii) and uses crucially the bounds (a) and (b).

It is difficult to overestimate the impact of this work in the well-posedness theory. It was simply a complete game changer. Versions of the spaces just described were in the literature before, in earlier works of Rauch and Reed [76] and M. Beals [3] dealing with propagation of singularities for solutions of semilinear wave equations, and were also implicit in the contemporary work of Klainerman and Machedon 55] on the local well-posedness of semilinear wave equations. However, the flexibility 
and universality of Bourgain's formulation of these spaces contributed decisively to their wide applicability in solving a large number of previously intractable problems in the work of many researchers.

We now turn to the work in $\left[\underline{8}\right.$, on $(\mathrm{gKdV})_{k}$, on $\mathbb{T}$. We will restrict ourselves to commenting on the results for $k=1$.

Theorem $2.2([8])$. ( $\mathrm{KdV})$ is locally well-posed on $L^{2}(\mathbb{T})$, with time of existence depending on $\left\|u_{0}\right\|_{L^{2}}$ and, hence by conservation of the $L^{2}$ norm, it is globally well-posed in $L^{2}(\mathbb{T})$.

The proof also proceeds by a contraction mapping argument, in spaces related to the ones given by (6) but adapted to the linear operator $\partial_{t}+\partial_{x}^{3}$. A first reduction is to the case of data of integral 0, that is whose zero Fourier coefficient vanishes. The space $X_{s}$ now has norm

$$
\|u\|_{s}=\left\{\sum_{n \in \mathbb{Z}, n \neq 0}|n|^{2 s} \int_{-\infty}^{+\infty}\left(1+\left|\lambda-n^{3}\right|\right)|\widehat{u}(n, \lambda)|^{2} d \lambda\right\}^{1 / 2}
$$

for $u$ defined for $(x, t) \in \mathbb{T}^{2}$ with mean in $x$ equal to 0 . The relevant version of (a), when $s=0$ is now

$\left(\mathrm{a}^{\prime}\right)$

$$
\|f\|_{L^{4}\left(\mathbb{T}^{2}\right)} \lesssim\left(\sum_{m, n \in \mathbb{Z}}\left(1+\left|n-m^{3}\right|\right)^{2 / 3}|\hat{f}(m, n)|^{2}\right)^{1 / 2} .
$$

A very important difference with (NLS) is the fact that there is a derivative in the nonlinearity and there is no linear local smoothing effect, as we mentioned earlier. Bourgain's crucial insight here was that there is a nonlinear smoothing effect, best captured by the function spaces introduced above. This is given in the following estimates: let $w(x, t)=\partial_{x}\left(u^{2}\right)(x, t)$, where we assume that $\int_{\mathbb{T}} u(x, t) d x=0$. Then, for $s \geq 0$,

$$
\begin{gathered}
\left(\sum_{n \neq 0}|n|^{2 s} \int \frac{|\widehat{w}(n, \lambda)|^{2}}{\left(1+\left|\lambda-n^{3}\right|\right)} d \lambda\right)^{1 / 2} \lesssim\|u\|_{X_{s}} \\
\left(\sum_{n \neq 0}|n|^{2 s}\left(\int \frac{|\widehat{w}(n, \lambda)|}{\left(1+\left|\lambda-n^{3}\right|\right)} d \lambda\right)^{2}\right)^{1 / 2} \lesssim\|u\|_{X_{s}} .
\end{gathered}
$$

It is through these estimates, controlling $\partial_{x}\left(u^{2}\right)$ by $u$, that we see this nonlinear smoothing effect, which is a consequence of the curvature of $\left(n, n^{3}\right)$.

Finally, also in 8 , Bourgain observed that this nonlinear smoothing effect also carries over to the case $x \in \mathbb{R}$, using the function spaces

$$
X_{b}^{s}=\left\{u(x, t):\left.\iint\left(1+\left|\lambda-\xi^{3}\right|\right)^{2 b} \cdot|1+| \xi\right|^{2 s}|\widehat{u}(\xi, \lambda)|^{2} d \xi d \lambda<\infty\right.
$$

$$
\text { where } \left.(\xi, \lambda) \in \mathbb{R}^{2}\right\} \text {. }
$$

He proved

Theorem $2.3\left([\underline{8})\right.$. (KdV) is globally well-posed in $L^{2}(\mathbb{R})$. 
Remark 2. By using a nonlinear smoothing effect and thus replacing $v \partial_{x} w$ in Remark 1 by $\partial_{x}\left(u^{2}\right)$, Bourgain bypassed the objection for improving $s>\frac{3}{4}$, given in Remark 1. To Ponce, Vega, and myself this was a shocking observation. Of course, this was just one of the many shocking observations made by Bourgain over the years! These works of Bourgain have been and continue to be remarkably influential.

Remark 3. Theorems 2.2 and 2.3 generated substantial interest in the question of finding the optimal $s$ for local well-posedness in each theorem. In [50], it was shown that local well-posedness for $\mathbb{T}$ holds for $s>-\frac{1}{2}$ and for $\mathbb{R}$ for $s>-\frac{3}{4}$, both by the contraction mapping principle. In 12 Bourgain observed that local well-posedness cannot be proved by the contraction mapping principle, for $s<-\frac{1}{2}$ on $\mathbb{T}$ and for $s<-\frac{3}{4}$ on $\mathbb{R}$. In [40] and [54] it was shown (independently) that local well-posedness holds in $H^{-\frac{1}{2}}(\mathbb{T})$ and $H^{-\frac{3}{4}}(\mathbb{R})$, by the contraction mapping principle, using a modification of the spaces $X_{b}^{s}$ introduced by Bourgain. That a modification of the spaces was needed was shown by Nakanishi, Takaoka, and Tsutsumi [72. Finally, local well-posedness was shown in $H^{-1}(\mathbb{T})$ by Kappeler and Topalov in [41] and by Killip and Visan in $H^{-1}(\mathbb{R})([53])$, using inverse scattering. These are the optimal spaces for local well-posedness in the scale of Sobolev spaces, as was shown by Molinet [69], 70.

3. A QUiCK SAMPLing OF SOME

OF THE OTHER GROUNDBREAKING CONTRIBUTIONS OF BOURGAIN TO NONLINEAR DISPERSIVE EQUATIONS

3.1. Gibbs measure associated to periodic (NLS). We again consider the (NLS) equation

$$
\left\{\begin{array}{l}
i \partial_{t} u+\Delta u \pm|u|^{p-1} u=0, p>1, u: \mathbb{T}^{d} \times \mathbb{R} \rightarrow \mathbb{C} \\
\left.u\right|_{t=0}=u_{0}
\end{array}\right.
$$

and recall the two conserved quantities, the mass

$$
M(u)=\int_{\mathbb{T}^{d}}|u|^{2} d x=M\left(u_{0}\right)
$$

and the Hamiltonian (the energy)

$$
H(u)=\frac{1}{2} \int_{\mathbb{T}^{d}}|\nabla u|^{2} d x \pm \frac{1}{p+1} \int_{\mathbb{T}^{d}}|u|^{p+1} d x=H\left(u_{0}\right) .
$$

If we set $\hat{u}(n, t)=a_{n}(t)+i b_{n}(t)$, we see that $u$ solves (NLS) if and only if $\dot{a}_{n}(t)=\frac{\partial H}{\partial b_{n}}$ and $\dot{b}_{n}(t)=-\frac{\partial H}{\partial a_{n}}, n \in \mathbb{Z}^{d}$. Thus, (NLS) can be viewed as an infinite-dimensional Hamiltonian system. If the Hamiltonian system is finite dimensional, say we consider $|n| \leq N$, then the Gibbs measure $d \mu$, given by

$$
d \mu=\frac{1}{Z_{N}} e^{-H\left(a_{n}, b_{n}\right)} \prod_{|n| \leq N} d a_{n} d b_{n},
$$

where $Z_{N}$ is a normalization constant, is well-defined and invariant with respect to the flow. In their paper [56], Lebowitz, Rose, and Speer were able to make sense 
of the Gibbs measure associated to (NLS) in $\mathbb{T}$, with $p=5$. They considered the formal expression

$$
d \mu=\frac{1}{Z} e^{-H\left(a_{n}, b_{n}\right)} \prod_{n \in \mathbb{Z}} d a_{n} d b_{n}
$$

by introducing first the Gaussian measure

$$
d \rho=\frac{1}{\tilde{Z}} e^{-\sum_{n}\left(1+n^{2}\right)\left(\left|a_{n}\right|^{2}+\left|b_{n}\right|^{2}\right)} \prod_{n} d a_{n} d b_{n},
$$

with support in $H^{s}(\mathbb{T}), s<\frac{1}{2}$, and they then proved that $d \mu$ is absolutely continuous with respect to $d \rho$. The questions they formulated follow.

(1) Is (NLS) on $\mathbb{T}$, with $p=5$, on $H^{s}(\mathbb{T}), 0<s<\frac{1}{2}$, well-defined for all times, at least for data in the support of the measure?

(2) Is $d \mu$ invariant with respect to the (NLS) flow?

In the paper [10, Bourgain answered both questions in the positive. To treat both issues, he used the locally well-posed result in $H^{s}, 0<s<\frac{1}{2}$, given in Theorem 2.1. and then used the invariance of the measure under the flow to establish global well-posedness almost surely $d \mu$.

In 11] Bourgain then treated a very challenging question along these lines: can one do this for the cubic (NLS) on $\mathbb{T}^{2}$, at least in the defocusing case? That is, for the equation

$$
i \partial_{t} u+\Delta u-|u|^{2} u=0, x \in \mathbb{T}^{2} .
$$

The existence of $d \mu$ in this case was due to Glimm and Jaffe [36, but $\operatorname{supp} \mu \subset$ $H^{s}\left(\mathbb{T}^{2}\right), s<0$, while Theorem 2.1 gives local well-posedness in $H^{s}\left(\mathbb{T}^{2}\right), s>0$.

Bourgain overcame this difficulty through another shocking breakthrough. He considered the following random data:

$$
u_{0}^{\omega}=\sum_{n \in \mathbb{Z}^{2}} \frac{g_{n}(\omega)}{\left(1+|n|^{2}\right)^{\frac{1}{2}}} e^{i n x},
$$

where the $\left\{g_{n}\right\}$ are identically distributed complex Gaussian random variables. Since $u_{0}^{\omega} \in H^{s}\left(\mathbb{T}^{2}\right), s<0, u_{0}^{\omega}$ belongs to the support of the Gibbs measure $\mu$. (We are going to ignore here the need for Wick-ordering the (NLS) equation here; see [11.) The key observation is that if $u$ is the (NLS) solution, $w(t)=u(t)-S(t) u_{0}^{\omega}$ is (almost surely in $\omega$ ) well-defined in $H^{\bar{s}}\left(\mathbb{T}^{2}\right)$, where $\bar{s}>0$, and one can then solve for $w$, to obtain a local-in-time solution. Finally, the local-in-time solution is extended globally in time, using the invariance of the Gibbs measure. This very influential paper led to the notion of probabilistic well-posedness in dispersive equations in works of Burq and Tzvelkov [20], T. Oh [73, and many others, including Bourgain and Bulut ([17, [18).

3.2. Bourgain's "high-low decomposition". In Theorem 1.1 the local-in-time result can be extended to a global-in-time one, in the case where the $H^{s}$ norm of the data is small, $s \geq s_{0}$. In the mass $\left(L^{2}\right)$ subcritical case, when $p-1<4 / d$ (that is when $\left.s_{0}<0\right)$, the problem is locally well-posed in $L^{2}$, and hence globally wellposed in $L^{2}$. When $p-1 \geq 4 / d$, in the focusing case (that is when the sign in front of the nonlinearity in (NLS) is negative, and hence the Hamiltonian does not have a definite sign), a sufficiently large smooth solution may blow up in finite time (see Glassey ([35]), Merle ([58], [59]), Bourgain and Wang ([19]), Merle and Raphaël (60], 61], 62, 63], 64), Raphaël (74, 65]), Merle, Raphaël, and Rodnianski 
([65), etc.). Also, if the nonlinearity is defocusing (that is, the sign in front of the nonlinear term in (NLS) is negative so that the conserved Hamiltonian

$$
H(u)=\frac{1}{2} \int|\nabla u|^{2}+\frac{1}{p+1} \int|u|^{p+1}
$$

controls $\int|\nabla u|^{2}$, and if $p-1<\frac{4}{d-2}$ (that is $s_{0}<1$ ) and hence the problem is energy subcritical), (NLS) is globally well-posed in the energy sphere $H^{1}\left(\mathbb{R}^{d}\right)$ by iterating the result in Theorem 1.1.

In 13. Bourgain developed a very general method to, in such circumstances, obtain global well-posedness below the energy norm. A sample result is

Theorem 3.1 ([13]). The problem

$$
\left\{\begin{array}{l}
i \partial_{t} u+\Delta u-u|u|^{2}=0 \\
\left.u\right|_{t=0}=u_{0} \in H^{s}\left(\mathbb{R}^{2}\right)
\end{array}\right.
$$

is globally well-posed for $s>\frac{3}{5}$. Moreover, the solution $u$ satisfies $u(t)-S(t) u_{0} \in$ $H^{1}\left(\mathbb{R}^{2}\right)$ for all $t$ (with a polynomial control in $|t|$ of the $H^{1}$ norm).

The general scheme of the method is as follows: First, one has to have a conserved quantity (say $I\left(u_{0}\right)$ ), such that $I\left(u_{0}\right)$ controls a certain $H^{s_{0}}$ norm. Next, one needs a local well-posedness result in $H^{s_{1}}$, for $s_{1}<s_{0}$, with the flow map satisfying $I\left(u(t)-S(t) u_{0}\right) \leq F\left(\left\|u_{0}\right\|_{H^{s_{1}}}\right)$, where $S(t)$ is the associated linear evolution, acting unitarily on all $H^{s}$ spaces. One then expects a global well-posedness result in $H^{s_{2}}$, for some $s_{1}<s_{2}<s_{0}$. In the theorem stated, $I$ is the Hamiltonian. One then splits, for some $T$ large and fixed, $u_{0}=u_{0,1}^{\left(N_{0}\right)}+u_{0,2}^{\left(N_{0}\right)}$, with $u_{0,1}^{(N)}=\int_{|\xi| \leq N_{0}} \hat{u}_{0}(\xi) e^{i x \cdot \xi} d \xi$, where $N_{0}=N_{0}(T)$ is to be chosen.

It is simple to see that $H\left(u_{0,1}^{\left(N_{0}\right)}\right) \lesssim N_{0}^{2(1-s)}$. One then solves the nonlinear problem with initial data $u_{0,1}^{\left(N_{0}\right)}$, for all times. If we choose the time interval $I=$ $[0, \delta]$, where $\delta=N_{0}^{-2(1-s)-\epsilon}$,

$$
\left\|u_{0,1}^{\left(N_{0}\right)}\right\|_{L^{4}\left(\mathbb{R}^{d} \times I\right)}=o(1) .
$$

If we let $u=u_{1}^{\left(N_{0}\right)}+v$, where $u_{1}^{\left(N_{0}\right)}$ is the global solution just mentioned, $v$ satisfies the difference equation

$$
\left\{\begin{array}{l}
i \partial_{t} v+\Delta v-2\left|u_{1}^{\left(N_{0}\right)}\right|^{2} v-\left(u_{1}^{\left(N_{0}\right)}\right)^{2} \bar{v}-\overline{\left(u_{1}^{\left(N_{0}\right)}\right)} v^{2}-2 u_{1}^{\left(N_{0}\right)}|v|^{2}-|v|^{2} v=0 \\
\left.v\right|_{t=0}=u_{0,2}^{\left(N_{0}\right)}
\end{array}\right.
$$

with $\left\|u_{0,2}^{\left(N_{0}\right)}\right\|_{L^{2}} \lesssim N_{0}^{-s} ;\left\|u_{0,2}^{\left(N_{0}\right)}\right\|_{H^{s}} \leq C$. One then gets, after calculations, $v=$ $S(t)\left(u_{0,2}^{\left(N_{0}\right)}\right)+w$, where $w(t) \in H^{1},\|w(t)\|_{L^{2}} \lesssim N_{0}^{-s}$, and $\|w(t)\|_{H^{1}} \lesssim N_{0}^{1-2 s+\epsilon}$.

Then, fixing $t_{1}=\delta$, we obtain $u\left(t_{1}\right)=u_{1}+v_{1}$, where $u_{1}=u_{1}^{\left(N_{0}\right)}\left(t_{1}\right)+w\left(t_{1}\right)$, $v_{1}=S\left(t_{1}\right)\left(u_{0,2}^{\left(N_{0}\right)}\right)$. Using the conservation of $H$, and the bounds for $w$, this yields

$$
H\left(u_{1}\right) \leq H\left(u_{0}\right)+C N_{0}^{2-3 s+\epsilon},
$$

while $v_{1}$ has the same properties as $u_{0,2}^{\left(N_{0}\right)}$. Iterating the procedure, to reach time $T$, we need a number of steps,

$$
\frac{T}{\delta} \simeq T \cdot N_{0}^{2(1-s)+\epsilon}
$$


Thus we need to ensure that

$$
T \cdot N_{0}^{2(1-s)+\epsilon} \cdot N_{0}^{2-3 s+\epsilon}<H\left(u_{0,1}^{\left(N_{0}\right)}\right) \approx N_{0}^{2(1-s)} .
$$

This can be achieved for $s>\frac{2}{3}$. A more elaborate argument gives $s>\frac{3}{5}$.

This method, as mentioned before, is very general, and has led to many global well-posedness results, due to many researchers, for instance in energy subcritical, defocusing problems. The method also stimulated the I-team (Colliander, Keel, Staffilani, Takaoka, and Tao) to develop the $I$-method to treat similar types of situations. The $I$-method has been extraordinarily successful (see for instance [25], [26], 27], 28, etc.).

Besides his interest in global well-posedness for defocusing, energy subcritical (NLS), Bourgain was very interested in corresponding global-in-time results for energy critical and supercritical (NLS). In the next section we will discuss Bourgain's work in the energy critical case. Understanding the global-in-time energysupercritical case was a problem that Bourgain considered very natural and intriguing. In [16, Bourgain conjectured the global existence of classical solutions, with smooth, well-localized data for defocusing energy supercritical (NLS). For years, this problem was considered out of reach. Recently, this conjecture was disproved for $d \geq 5$ in the spectacular series of papers by Merle, Raphaël, Rodnianski, and Szeftel ([66, 67]), who also were able to obtain corresponding results for the compressible Euler and Navier-Stokes flows 68.

3.3. Bourgain's work on the defocusing energy critical (NLS). In the remarkable paper [14, Bourgain considered the defocusing, energy critical (NLS)

$$
\left\{\begin{array}{l}
i \partial_{t} u+\Delta u-|u|^{\frac{4}{d-2}} u=0, d \geq 3 \\
\left.u\right|_{t=0}=u_{0} \in H^{1}\left(\mathbb{R}^{d}\right) .
\end{array}\right.
$$

Theorem 3.2. (7) is globally well-posed for $u_{0}$ radial, when $d=3,4$. Moreover, higher regularity of $u_{0}$ is preserved for all times.

Remark 4 . The result was proved independently by Grillakis 39, when $d=3$. It was extended to all $d \geq 3$, still under $u_{0}$ radial, by Tao in 2005 .

Remark 5. In addition to global well-posedness, Bourgain established scattering, that is to say, there exist $u_{0}^{ \pm} \in H^{1}\left(\mathbb{R}^{d}\right)$, radial such that

$$
\lim _{t \rightarrow \pm \infty}\left\|u(t)-S(t)\left(u_{0}^{ \pm}\right)\right\|_{H^{1}\left(\mathbb{R}^{d}\right)}=0 .
$$

Remark 6 . The corresponding result for the defocusing energy critical nonlinear wave equation

$$
\left\{\begin{array}{l}
\partial_{t}^{2} u-\Delta u+|u|^{\frac{4}{d-2}} u=0 \\
\left.u\right|_{t=0}=u_{0} \in H^{1}\left(\mathbb{R}^{d}\right) \\
\left.\partial_{t} u\right|_{t=0}=u_{1} \in L^{2}\left(\mathbb{R}^{d}\right)
\end{array}\right.
$$

were established by Struwe [84 in the radial case, by Grillakis [37, 38, in the nonradial case (see also [79], [80]), with scattering being obtained by Bahouri and Shatah in [2]. The key idea was to use the Morawetz identity [71, which for the wave equation has energy critical scaling, combined with finite speed of propagation (another important feature of the wave equation) to prevent energy concentration. 
For the proof of Theorem 3.2, when $d=3$, the starting point is to show that if

$$
\int_{0}^{T_{\star}} \int_{\mathbb{R}^{3}}|u(x, t)|^{10} d x d t<\infty,
$$

where $T_{\star}$ is the final time of existence of $u$, then $T_{\star}=\infty$ and $u$ scatters. This fact is now referred to as the standard finite time blow-up criterion. In order to achieve (8), Bourgain's idea was to do so by induction on the size of the Hamiltonian of $u_{0}$, and show that

$$
\|u\|_{L_{x}^{10} L_{\left[0, T_{\star}\right]}^{10}} \leq M\left(H\left(u_{0}\right)\right)
$$

for some function $M$. It is easy to show from the proof of the local well-posedness result (since $\left.\left\|u_{0}\right\|_{H^{1}} \lesssim H\left(u_{0}\right)\right)$ that this is the case if $H\left(u_{0}\right)$ is small. Arguing by contradiction, one assumes that

$$
\|u\|_{L_{x}^{10} L_{\left[0, T_{\star}\right]}^{10}}>M
$$

for some $M$ large, and that $\|v\|_{L_{x}^{10} L_{t}^{10}}<M_{1}$, whenever

$$
\left\{\begin{array}{l}
i \partial_{t} v+\Delta v-|v|^{4} v=0 \\
\left.v\right|_{t=0}=v_{0}
\end{array}\right.
$$

provided $H\left(v_{0}\right)<H\left(u_{0}\right)-\eta^{4}$, for some small $\eta$ (depending only on $H\left(u_{0}\right)$ ), and then one reaches a contradiction for large $M$.

In order to reach this contradiction, Bourgain introduced a modification of the Morawetz estimate for the Schrödinger equation, due to Lin and Strauss [57]. Comparing Theorem 3.2 with the earlier work on the wave equation by Grillakis mentioned in Remark 6, key difficulties are the infinite speed of propagation and the unfavorable scaling of the estimate in [57. This is addressed in the following.

Proposition 1. Let $u$ be a solution of (7) in the energy space on a time interval I on which (7) is well-posed in the energy space. Then,

$$
\int_{I} \int_{|x|<|I|^{1 / 2}} \frac{|u(x, t)|^{6}}{|x|} d x d t \leq C H\left(u_{0}\right)|I|^{1 / 2} .
$$

It is in the application of this proposition (which allows one to handle energy concentration) that the radial hypothesis is used. The details of the proof are intricate. The induction on energy used in the proof is an audacious idea, which has been extremely influential. In [29] the I-team (Colliander, Keel, Staffilani, Takaoka, and Tao) in a major breakthrough, extended the $d=3$ result in Theorem 3.2 to the nonradial case. An important ingredient of their proof is the introduction of an interaction Morawetz inequality, a version of Proposition 1, in which the origin is not a privileged point. This was extended to $d=4$ by Ryckman and Visan [77] and to $d \geq 5$ by Visan [89. Later on a new method, dubbed the concentrationcompactness/rigidity theorem method was introduced in [46, 47], 48, which is very flexible, and which could also treat focusing problems under sharp size conditions. This method also led to many more developments in these types of problems in the works of many researchers. For a proof of Theorem 3.2 and its nonradial version in [29] using this new method, see the work of Killip and Visan [52]. 


\section{Conclusion}

The work of Jean Bourgain transformed the field of nonlinear dispersive equations by settling old conjectures, introducing new methods and ideas, and posing important problems. The works briefly described in this note are just a small (hopefully representative) sample of Bourgain's influential contributions to this field. They will continue to inspire researchers for generations to come.

\section{ACKNOWLEDGMENTS}

The author is very grateful to Gigliola Staffilani, for sharing with him the slides of her lecture at the meeting "Honoring the Life and Work of Jean Bourgain" held at IAS, Princeton on May 31-June 01, 2019. In particular, the presentation in Section 4.1 follows very closely her exposition.

\section{ABout THE AUthor}

Carlos E. Kenig is the Louis Block Distinguished Service Professor at the University of Chicago. He specializes in harmonic analysis and partial differential equations.

\section{REFERENCES}

[1] M. J. Ablowitz and P. A. Clarkson, Solitons, nonlinear evolution equations and inverse scattering, London Mathematical Society Lecture Note Series, vol. 149, Cambridge University Press, Cambridge, 1991. MR.1149378

[2] H. Bahouri and J. Shatah, Decay estimates for the critical semilinear wave equation (English, with English and French summaries), Ann. Inst. H. Poincaré Anal. Non Linéaire 15 (1998), no. 6, 783-789, DOI 10.1016/S0294-1449(99)80005-5. MR:1650958

[3] M. Beals, Self-spreading and strength of singularities for solutions to semilinear wave equations, Ann. of Math. (2) 118 (1983), no. 1, 187-214, DOI 10.2307/2006959. MR707166

[4] B. Birnir, C. E. Kenig, G. Ponce, N. Svanstedt, and L. Vega, On the ill-posedness of the IVP for the generalized Korteweg-de Vries and nonlinear Schrödinger equations, J. London Math. Soc. (2) 53 (1996), no. 3, 551-559, DOI 10.1112/jlms/53.3.551. MR1396718

[5] J. L. Bona and R. Smith, The initial-value problem for the Korteweg-de Vries equation, Philos. Trans. Roy. Soc. London Ser. A 278 (1975), no. 1287, 555-601, DOI 10.1098/rsta.1975.0035. MR 385355

[6] J. Bona and R. Scott, Solutions of the Korteweg-de Vries equation in fractional order Sobolev spaces, Duke Math. J. 43 (1976), no. 1, 87-99. MR393887

[7] J. Bourgain, Fourier transform restriction phenomena for certain lattice subsets and applications to nonlinear evolution equations. I. Schrödinger equations, Geom. Funct. Anal. 3 (1993), no. 2, 107-156, DOI 10.1007/BF01896020. MR1209299

[8] J. Bourgain, Fourier transform restriction phenomena for certain lattice subsets and applications to nonlinear evolution equations. II. The KdV-equation, Geom. Funct. Anal. 3 (1993), no. 3, 209-262, DOI 10.1007/BF01895688. MR1215780

[9] J. Bourgain, Exponential sums and nonlinear Schrödinger equations, Geom. Funct. Anal. 3 (1993), no. 2, 157-178, DOI 10.1007/BF01896021. MR1209300

[10] J. Bourgain, Periodic nonlinear Schrödinger equation and invariant measures, Comm. Math. Phys. 166 (1994), no. 1, 1-26. MR 1309539

[11] J. Bourgain, Invariant measures for the 2D-defocusing nonlinear Schrödinger equation, Comm. Math. Phys. 176 (1996), no. 2, 421-445. MR1374420

[12] J. Bourgain, Periodic Korteweg de Vries equation with measures as initial data, Selecta Math. (N.S.) 3 (1997), no. 2, 115-159, DOI 10.1007/s000290050008. MR.1466164

[13] J. Bourgain, Refinements of Strichartz' inequality and applications to $2 D-N L S$ with critical nonlinearity, Internat. Math. Res. Notices 5 (1998), 253-283, DOI 10.1155/S1073792898000191. MR1616917 
[14] J. Bourgain, Global wellposedness of defocusing critical nonlinear Schrödinger equation in the radial case, J. Amer. Math. Soc. 12 (1999), no. 1, 145-171, DOI 10.1090/S0894-0347-9900283-0. MR 1626257

[15] J. Bourgain, Global solutions of nonlinear Schrödinger equations, American Mathematical Society Colloquium Publications, vol. 46, American Mathematical Society, Providence, RI, 1999. MR 1691575

[16] J. Bourgain, Problems in Hamiltonian PDE's, Geom. Funct. Anal. Special Volume (2000), 32-56, DOI 10.1007/978-3-0346-0422-2_2. GAFA 2000 (Tel Aviv, 1999). MR1826248

[17] J. Bourgain and A. Bulut, Almost sure global well posedness for the radial nonlinear Schrödinger equation on the unit ball I: the 2D case, Ann. Inst. H. Poincaré Anal. Non Linéaire 31 (2014), no. 6, 1267-1288, DOI 10.1016/j.anihpc.2013.09.002. MR3280067

[18] J. Bourgain and A. Bulut, Almost sure global well-posedness for the radial nonlinear Schrödinger equation on the unit ball II: the $3 d$ case, J. Eur. Math. Soc. (JEMS) 16 (2014), no. 6, 1289-1325, DOI 10.4171/JEMS/461. MR3226743

[19] J. Bourgain and W. Wang, Construction of blowup solutions for the nonlinear Schrödinger equation with critical nonlinearity, Ann. Scuola Norm. Sup. Pisa Cl. Sci. (4) 25 (1997), no. 1-2, 197-215 (1998). Dedicated to Ennio De Giorgi. MR 1655515

[20] N. Burq and N. Tzvetkov, Random data Cauchy theory for supercritical wave equations. II. A global existence result, Invent. Math. 173 (2008), no. 3, 477-496, DOI 10.1007/s00222-0080123-0. MR2425134

[21] L. Carleson, Some analytic problems related to statistical mechanics, Euclidean harmonic analysis (Proc. Sem., Univ. Maryland, College Park, Md., 1979), Lecture Notes in Math., vol. 779, Springer, Berlin, 1980, pp. 5-45. MR576038

[22] T. Cazenave and F. B. Weissler, The Cauchy problem for the critical nonlinear Schrödinger equation in $H^{s}$, Nonlinear Anal. 14 (1990), no. 10, 807-836, DOI 10.1016/0362546X(90)90023-A. MR 1055532

[23] R. R. Coifman and Y. Meyer, Au delà des opérateurs pseudo-différentiels (French), Astérisque, vol. 57, Société Mathématique de France, Paris, 1978. With an English summary. MR.518170

[24] R. R. Coifman and Y. Meyer, Nonlinear harmonic analysis, operator theory and P.D.E, Beijing lectures in harmonic analysis (Beijing, 1984), Ann. of Math. Stud., vol. 112, Princeton Univ. Press, Princeton, NJ, 1986, pp. 3-45. MR864370

[25] J. Colliander, M. Keel, G. Staffilani, H. Takaoka, and T. Tao, A refined global well-posedness result for Schrödinger equations with derivative, SIAM J. Math. Anal. 34 (2002), no. 1, 64-86, DOI 10.1137/S0036141001394541. MR1950826

[26] J. Colliander, M. Keel, G. Staffilani, H. Takaoka, and T. Tao, Sharp global well-posedness for $K d V$ and modified $K d V$ on $\mathbb{R}$ and $\mathbb{T}$, J. Amer. Math. Soc. 16 (2003), no. 3, 705-749, DOI 10.1090/S0894-0347-03-00421-1. MR.1969209

[27] J. Colliander, M. Keel, G. Staffilani, H. Takaoka, and T. Tao, Global existence and scattering for rough solutions of a nonlinear Schrödinger equation on $\mathbb{R}^{3}$, Comm. Pure Appl. Math. $\mathbf{5 7}$ (2004), no. 8, 987-1014, DOI 10.1002/cpa.20029. MR2053757

[28] J. Colliander, M. Keel, G. Staffilani, H. Takaoka, and T. Tao, Multilinear estimates for periodic KdV equations, and applications, J. Funct. Anal. 211 (2004), no. 1, 173-218, DOI 10.1016/S0022-1236(03)00218-0. MR2054622

[29] J. Colliander, M. Keel, G. Staffilani, H. Takaoka, and T. Tao, Global well-posedness and scattering for the energy-critical nonlinear Schrödinger equation in $\mathbb{R}^{3}$, Ann. of Math. (2) 167 (2008), no. 3, 767-865, DOI 10.4007/annals.2008.167.767. MR2415387

[30] P. Constantin and J.-C. Saut, Local smoothing properties of dispersive equations, J. Amer. Math. Soc. 1 (1988), no. 2, 413-439, DOI 10.2307/1990923. MR928265

[31] B. E. J. Dahlberg and C. E. Kenig, A note on the almost everywhere behavior of solutions to the Schrödinger equation, Harmonic analysis (Minneapolis, Minn., 1981), Lecture Notes in Math., vol. 908, Springer, Berlin-New York, 1982, pp. 205-209. MR654188

[32] J. Ginibre and G. Velo, On a class of nonlinear Schrödinger equations. I. The Cauchy problem, general case, J. Functional Analysis 32 (1979), no. 1, 1-32, DOI 10.1016/00221236(79)90076-4. MR533218

[33] J. Ginibre and G. Velo, On a class of nonlinear Schrödinger equations. III. Special theories in dimensions 1, 2 and 3, Ann. Inst. H. Poincaré Sect. A (N.S.) 28 (1978), no. 3, 287-316. MR 498408 
[34] J. Ginibre and G. Velo, The global Cauchy problem for the nonlinear Schrödinger equation revisited (English, with French summary), Ann. Inst. H. Poincaré Anal. Non Linéaire 2 (1985), no. 4, 309-327. MR801582

[35] R. T. Glassey, On the blowing up of solutions to the Cauchy problem for nonlinear Schrödinger equations, J. Math. Phys. 18 (1977), no. 9, 1794-1797, DOI 10.1063/1.523491. MR 460850

[36] J. Glimm and A. Jaffe, Quantum physics: A functional integral point of view, 2nd ed., Springer-Verlag, New York, 1987. MR887102

[37] M. G. Grillakis, Regularity and asymptotic behaviour of the wave equation with a critical nonlinearity, Ann. of Math. (2) 132 (1990), no. 3, 485-509, DOI 10.2307/1971427. MR 1078267

[38] M. G. Grillakis, Regularity for the wave equation with a critical nonlinearity, Comm. Pure Appl. Math. 45 (1992), no. 6, 749-774, DOI 10.1002/cpa.3160450604. MR.1162370

[39] M. G. Grillakis, On nonlinear Schrödinger equations, Comm. Partial Differential Equations 25 (2000), no. 9-10, 1827-1844, DOI 10.1080/03605300008821569. MR.1778782

[40] Z. Guo, Global well-posedness of Korteweg-de Vries equation in $H^{-3 / 4}(\mathbb{R})$ (English, with English and French summaries), J. Math. Pures Appl. (9) 91 (2009), no. 6, 583-597, DOI 10.1016/j.matpur.2009.01.012. MR2531556

[41] T. Kappeler and P. Topalov, Global wellposedness of $K d V$ in $H^{-1}(\mathbb{T}, \mathbb{R})$, Duke Math. J. 135 (2006), no. 2, 327-360, DOI 10.1215/S0012-7094-06-13524-X. MR2267286

[42] T. Kappeler and P. Topalov, Global well-posedness of $m K d V$ in $L^{2}(\mathbb{T}, \mathbb{R})$, Comm. Partial Differential Equations 30 (2005), no. 1-3, 435-449, DOI 10.1081/PDE-200050089. MR2131061

[43] T. Kato, On the Korteweg-de Vries equation, Manuscripta Math. 28 (1979), no. 1-3, 89-99, DOI 10.1007/BF01647967. MR535697

[44] T. Kato, On the Cauchy problem for the (generalized) Korteweg-de Vries equation, Studies in Applied Mathematics, Adv. Math. Suppl. Stud., vol. 8, Academic Press, New York, 1983, pp. 93-128. MR759907

[45] T. Kato, On nonlinear Schrödinger equations (English, with French summary), Ann. Inst. H. Poincaré Phys. Théor. 46 (1987), no. 1, 113-129. MR877998

[46] C. E. Kenig and F. Merle, Global well-posedness, scattering and blow-up for the energycritical, focusing, non-linear Schrödinger equation in the radial case, Invent. Math. 166 (2006), no. 3, 645-675, DOI 10.1007/s00222-006-0011-4. MR2257393

[47] C. E. Kenig and F. Merle, Global well-posedness, scattering and blow-up for the energycritical focusing non-linear wave equation, Acta Math. 201 (2008), no. 2, 147-212, DOI 10.1007/s11511-008-0031-6. MR2461508

[48] C. E. Kenig and F. Merle, Scattering for $\dot{H}^{1 / 2}$ bounded solutions to the cubic, defocusing NLS in 3 dimensions, Trans. Amer. Math. Soc. 362 (2010), no. 4, 1937-1962, DOI 10.1090/S00029947-09-04722-9. MR2574882

[49] C. E. Kenig, G. Ponce, and L. Vega, Well-posedness and scattering results for the generalized Korteweg-de Vries equation via the contraction principle, Comm. Pure Appl. Math. 46 (1993), no. 4, 527-620, DOI 10.1002/cpa.3160460405. MR.1211741

[50] C. E. Kenig, G. Ponce, and L. Vega, A bilinear estimate with applications to the KdV equation, J. Amer. Math. Soc. 9 (1996), no. 2, 573-603, DOI 10.1090/S0894-0347-96-00200-7. MR.1329387

[51] C. E. Kenig, G. Ponce, and L. Vega, On the ill-posedness of some canonical dispersive equations, Duke Math. J. 106 (2001), no. 3, 617-633, DOI 10.1215/S0012-7094-01-106388. MR 1813239

[52] R. Killip and M. Vişan, Global well-posedness and scattering for the defocusing quintic NLS in three dimensions, Anal. PDE 5 (2012), no. 4, 855-885, DOI 10.2140/apde.2012.5.855. MR 3006644

[53] R. Killip and M. Vişan, $K d V$ is well-posed in $H^{-1}$, Ann. of Math. (2) 190 (2019), no. 1, 249-305, DOI 10.4007/annals.2019.190.1.4. MR3990604

[54] N. Kishimoto, Well-posedness of the Cauchy problem for the Korteweg-de Vries equation at the critical regularity, Differential Integral Equations 22 (2009), no. 5-6, 447-464. MR2501679

[55] S. Klainerman and M. Machedon, Space-time estimates for null forms and the local existence theorem, Comm. Pure Appl. Math. 46 (1993), no. 9, 1221-1268, DOI 10.1002/cpa.3160460902. MR.1231427 
[56] J. L. Lebowitz, H. A. Rose, and E. R. Speer, Statistical mechanics of the nonlinear Schrödinger equation, J. Statist. Phys. 50 (1988), no. 3-4, 657-687, DOI 10.1007/BF01026495. MR.939505

[57] J. E. Lin and W. A. Strauss, Decay and scattering of solutions of a nonlinear Schrödinger equation, J. Functional Analysis 30 (1978), no. 2, 245-263, DOI 10.1016/0022-1236(78)900733. MR 515228

[58] F. Merle, Determination of blow-up solutions with minimal mass for nonlinear Schrödinger equations with critical power, Duke Math. J. 69 (1993), no. 2, 427-454, DOI 10.1215/S00127094-93-06919-0. MR 1203233

[59] F. Merle, Construction of solutions with exactly $k$ blow-up points for the Schrödinger equation with critical nonlinearity, Comm. Math. Phys. 129 (1990), no. 2, 223-240. MR.1048692

[60] F. Merle and P. Raphael, Sharp upper bound on the blow-up rate for the critical nonlinear Schrödinger equation, Geom. Funct. Anal. 13 (2003), no. 3, 591-642, DOI 10.1007/s00039003-0424-9. MR 1995801

[61] F. Merle and P. Raphael, On universality of blow-up profile for $L^{2}$ critical nonlinear Schrödinger equation, Invent. Math. 156 (2004), no. 3, 565-672, DOI 10.1007/s00222-0030346-z. MR2061329

[62] F. Merle and P. Raphael, Profiles and quantization of the blow up mass for critical nonlinear Schrödinger equation, Comm. Math. Phys. 253 (2005), no. 3, 675-704, DOI 10.1007/s00220004-1198-0. MR2116733

[63] F. Merle and P. Raphael, The blow-up dynamic and upper bound on the blow-up rate for critical nonlinear Schrödinger equation, Ann. of Math. (2) 161 (2005), no. 1, 157-222, DOI 10.4007/annals.2005.161.157. MR2150386

[64] F. Merle and P. Raphael, On a sharp lower bound on the blow-up rate for the $L^{2}$ critical nonlinear Schrödinger equation, J. Amer. Math. Soc. 19 (2006), no. 1, 37-90, DOI 10.1090/S0894-0347-05-00499-6. MR2169042

[65] F. Merle, P. Raphaël, and I. Rodnianski, Type II blow up for the energy supercritical NLS, Camb. J. Math. 3 (2015), no. 4, 439-617, DOI 10.4310/CJM.2015.v3.n4.a1. MR3435273

[66] F. Merle, P. Raphaël, I. Rodnianski, and J. Szeftel, On smooth self similar solutions to the compressible Euler equations, arXiv:1912.10998

[67] F. Merle, P. Raphaël, I. Rodnianski, and J. Szeftel, On blow-up for the energy super critical defocusing non linear Schrödinger equations, arXiv:1912.11009.

[68] F. Merle, P. Raphaël, I. Rodnianski, and J. Szeftel, On the implosion of a three dimensional compressible fluid, arXiv:1912.11009

[69] L. Molinet, A note on ill posedness for the KdV equation, Differential Integral Equations 24 (2011), no. 7-8, 759-765. MR2830706

[70] L. Molinet, Sharp ill-posedness results for the $K d V$ and $m K d V$ equations on the torus, Adv. Math. 230 (2012), no. 4-6, 1895-1930, DOI 10.1016/j.aim.2012.03.026. MR2927357

[71] C. S. Morawetz, Notes on time decay and scattering for some hyperbolic problems, Society for Industrial and Applied Mathematics, Philadelphia, Pa., 1975. Regional Conference Series in Applied Mathematics, No. 19. MR0492919

[72] K. Nakanishi, H. Takaoka, and Y. Tsutsumi, Counterexamples to bilinear estimates related with the KdV equation and the nonlinear Schrödinger equation, Methods Appl. Anal. 8 (2001), no. 4, 569-578, DOI 10.4310/MAA.2001.v8.n4.a7. IMS Conference on Differential Equations from Mechanics (Hong Kong, 1999). MR.1944182

[73] T. Oh, Invariant Gibbs measures and a.s. global well posedness for coupled KdV systems, Differential Integral Equations 22 (2009), no. 7-8, 637-668. MR2532115

[74] P. Raphael, Stability of the log-log bound for blow up solutions to the critical non linear Schrödinger equation, Math. Ann. 331 (2005), no. 3, 577-609, DOI 10.1007/s00208-0040596-0. MR2122541

[75] P. Raphaël, Existence and stability of a solution blowing up on a sphere for an $L^{2}$ supercritical nonlinear Schrödinger equation, Duke Math. J. 134 (2006), no. 2, 199-258, DOI 10.1215/S0012-7094-06-13421-X. MR2248831

[76] J. Rauch and M. Reed, Nonlinear microlocal analysis of semilinear hyperbolic systems in one space dimension, Duke Math. J. 49 (1982), no. 2, 397-475. MR659948

[77] E. Ryckman and M. Visan, Global well-posedness and scattering for the defocusing energycritical nonlinear Schrödinger equation in $\mathbb{R}^{1+4}$, Amer. J. Math. 129 (2007), no. 1, 1-60, DOI 10.1353/ajm.2007.0004. MR.2288737 
[78] I. Segal, Space-time decay for solutions of wave equations, Advances in Math. 22 (1976), no. 3, 305-311, DOI 10.1016/0001-8708(76)90097-9. MR492892

[79] J. Shatah and M. Struwe, Regularity results for nonlinear wave equations, Ann. of Math. (2) 138 (1993), no. 3, 503-518, DOI 10.2307/2946554. MR.1247991

[80] J. Shatah and M. Struwe, Well-posedness in the energy space for semilinear wave equations with critical growth, Internat. Math. Res. Notices 7 (1994), 303ff., approx. 7 pp., DOI 10.1155/S1073792894000346. MR.1283026

[81] E. M. Stein, Harmonic analysis: real-variable methods, orthogonality, and oscillatory integrals, Princeton Mathematical Series, vol. 43, Princeton University Press, Princeton, NJ, 1993. With the assistance of Timothy S. Murphy; Monographs in Harmonic Analysis, III. MR.1232192

[82] P. Sjölin, Regularity of solutions to the Schrödinger equation, Duke Math. J. 55 (1987), no. 3, 699-715, DOI 10.1215/S0012-7094-87-05535-9. MR.904948

[83] R. S. Strichartz, Restrictions of Fourier transforms to quadratic surfaces and decay of solutions of wave equations, Duke Math. J. 44 (1977), no. 3, 705-714. MR512086

[84] M. Struwe, Globally regular solutions to the $u^{5}$ Klein-Gordon equation, Ann. Scuola Norm. Sup. Pisa Cl. Sci. (4) 15 (1988), no. 3, 495-513 (1989). MR1015805

[85] Y. Tsutsumi, $L^{2}$-solutions for nonlinear Schrödinger equations and nonlinear groups, Funkcial. Ekvac. 30 (1987), no. 1, 115-125. MR915266

[86] P. A. Tomas, A restriction theorem for the Fourier transform, Bull. Amer. Math. Soc. 81 (1975), 477-478, DOI 10.1090/S0002-9904-1975-13790-6. MR358216

[87] L. Vega, Schrödinger equations: pointwise convergence to the initial data, Proc. Amer. Math. Soc. 102 (1988), no. 4, 874-878, DOI 10.2307/2047326. MR934859

[88] I. M. Vinogradov, The method of trigonometrical sums in the theory of numbers, Interscience Publishers, London and New York, 1954. Translated, revised and annotated by K. F. Roth and Anne Davenport. MR0062183

[89] M. Visan, The defocusing energy-critical nonlinear Schrödinger equation in higher dimensions, Duke Math. J. 138 (2007), no. 2, 281-374, DOI 10.1215/S0012-7094-07-13825-0. MR.2318286

University of ChicAgo, ChicAgo, Illinois

Email address: cek@math.uchicago.edu 